\title{
Evolution and structure of $5 S$ rDNA loci in allotetraploid Nicotiana tabacum and its putative parental species
}

\author{
J Fulneček ${ }^{1}, \mathrm{KY}$ Lim$^{2}$, AR Leitch ${ }^{2}$, A Kovař́ík ${ }^{1}$ and R Matyášek ${ }^{1}$ \\ ${ }^{1}$ Institute of Biophysics, Academy of Sciences of the Czech Republic, CZ-61265 Brno, Czech Republic; ${ }^{2}$ School of Biological Sciences, \\ Queen Mary, University of London, London E1 4NS, UK
}

\begin{abstract}
Nicotiana tabacum (tobacco) is an allotetraploid derived from ancestors of the modern diploids, $N$. sylvestris and $N$. tomentosiformis. We identified and characterized two distinct families of $5 S$ ribosomal DNA (rDNA) in N. tabacum; one family had an average $431 \mathrm{bp}$ unit length and the other a 646 bp unit length. In the diploid species, $N$. sylvestris and $N$. tomentosiformis, the 5S rDNA unit lengths are $431 \mathrm{bp}$ and 644 bp respectively. The non-coding spacer sequence of the short unit in tobacco had high sequence homology to the spacer of $N$. sylvestris $5 S$ rDNA, while the longer spacer of tobacco had high homology with the $5 S$ spacer of $N$. tomentosiformis. This suggests that the two $5 \mathrm{~S}$ families in tobacco have their origin in the diploid ancestors. The longer spacer sequence had a GC rich sub-region (called the T-genome sub-region) that was absent in the short spacer. Pulsed field gel analysis and fluorescent in situ hybridization to tobacco metaphase chromosomes showed that the two families of
\end{abstract}

5S rDNA units are spatially separate at two chromosomal loci, on chromosomes S8 (short family) and T8 (long family). The repeat copy number at each chromosomal locus showed heterogeneity between different tobacco cultivars, with a tendency for a decrease in the copy number of one family to be compensated by an increase in the copy number of the second family. Sequence analysis reveals there is as much diversity in $5 \mathrm{~S}$ family units within the diploid species as there is within the $T$ and S-genome $5 S$ family units respectively, suggesting $5 S$ diversification within each family had occurred before tobacco speciation. There is no evidence of interlocus homogenization of the two $5 \mathrm{~S}$ families in tobacco. This is therefore substantially different to 18-26S rDNA where interlocus gene conversion has substantially influenced most sequences of $\mathrm{S}$ and $\mathrm{T}$ genome origin; possible reasons are discussed.

Heredity (2002) 88, 19-25. DOI: 10.1038/sj/hdy/6800001

Keywords: allopolyploids; 5S rRNA; tobacco; gene conversion; evolution

\section{Introduction}

Nicotiana tabacum (tobacco) is a 5-6 million year old natural amphidiploid derived from ancestors of $N$. sylvestris, the maternal $S$ genome donor, and $N$. tomentosiformis the paternal T genome donor (Goodspeed, 1954; Okamuro and Goldberg, 1985; Parokonny and Kenton, 1995; Lim et al, 2000b). Comparisons of the tobacco genome with the genomes of modern diploid relatives have revealed several contrasting evolutionary patterns. Evolution of telomere repeat lengths in tobacco does not show an additive pattern to those found in N. sylvestris and N. tomentosiformis, but instead a tobacco-specific pattern (Kovař́k et al, 1996). Numerous studies based on genomic in situ hybridization showed clear discrimination of $S$ and $T$ genome chromosomes, with several macroscopic S/T or $\mathrm{T} / \mathrm{S}$ translocations involving, for example, the subtelomeric HRS60 repetitive sequence (Kenton et al, 1993; Moscone et al, 1996; Lim et al, 2000b). Recombination between $\mathrm{S}$ and $\mathrm{T}$ genomes has also been identified in members of

Correspondence: R Matyášek, Institute of Biophysics, Academy of Sciences of the Czech Republic, Královopolská 135, CZ-612 65 Brno, Czech Republic.E-mail: matyasek@ibp.cz

Received 7 September 2001; accepted 18 September 2001 the 1,3 $\beta$-glucanase gene family (Sperisen et al, 1991). But perhaps the best characterized $\mathrm{S}$ and $\mathrm{T}$ genome interaction is for the $18-26 S$ rDNA loci, where gene conversion has homogenized the $N$. tomentosiformis-type units and eliminated most units from the $N$. sylvestris parent (Kovařík et al, 1996; Volkov et al, 1999). This 18-26S rDNA gene conversion has not gone to completion, and it is possible that heavily methylated 18-26S rDNA units may not be as vulnerable to conversion as undermethylated units (Lim et al, 2000a).

Previously, two 5S rDNA families in N. tabacum were identified that differed in the length of the non-coding spacer sequence (Fulneček et al, 1998). Nicotiana rustica, another natural Nicotiana allotetraploid, also contains two $5 S$ rDNA families that also differ in the length of the intergenic spacers (Venkateswarlu et al, 1991). It is possible that the two $5 S$ rDNA families in N. tabacum and $N$. rustica have their origins in the diploid progenitor species. Certainly in the Triticeae (Scoles et al, 1988) and in the Gossypium allopolyploids, G. hirsutum and G. mustelinum (Cronn et al, 1996), two 5S rDNA families have been found that remained closely similar to the diploid progenitor species. In this paper we extend our analysis of $5 S$ rDNA families in tobacco to determine: (i) the organization, location and copy numbers of the two 5S families; 
(ii) whether the two families do indeed have their origin in the diploid progenitor species; and (iii) if gene conversion has influenced 5S evolution, as has occurred for 18$26 S$ rDNA. We employed sequencing of multiple clones from each species, and a distance tree was constructed. Using in situ hybridization and pulsed field gel electrophoresis (PFGE), the chromosomal distribution and large-scale structure of the $5 S$ gene families were studied, and the numbers and distributions of sites compared with tobacco's diploid relatives.

\section{Materials and methods}

\section{Plant material}

The following plant materials were used: Nicotiana tabacum L. cv. Vielblättriger, cv. Samsun, cv. Petite Havana SR-1, cv. 95-55, cv. Wisconsin 38, a callus of cv. Vielblättriger and suspension cultures, TBY-2 (Nagata et al, 1992) and VBI-0 (Opatrný and Opatrná, 1976), N. tomentosiformis Goodsp., N. otophora Griseb. and N. sylvestris Speg.

\section{Cloning and sequencing}

The intergenic spacer sequences of $5 S$ rDNA from $N$. tabacum, N. otophora, N. sylvestris and N. tomentosiformis were amplified using primers derived from the N. tabacum 5S rRNA genic sequence (Fulneček et al, 1998): 5SLF 5'-CCT GGG AAT TCC TCG TGT T, and 5SLR 5'TGC GTT AAA GCT TGT ATG ATC GCA T. EcoRI and HindIII restriction sites were included at the $5^{\prime}$ ends for cloning purposes. PCR conditions were as follows: $1 \mu \mathrm{g}$ total genomic DNA, $0.5 \mu \mathrm{M}$ each primer, $0.2 \mathrm{mM}$ each dNTP, $1 \times$ cloned Pfu DNA polymerase buffer, $2.5 \mathrm{U} P f u$ DNA polymerase (Stratagene) in a total volume of $100 \mu \mathrm{l}$. After denaturation at $94^{\circ} \mathrm{C}$ for $5 \mathrm{~min}$, the polymerase was added and 20 cycles $\left(94^{\circ} \mathrm{C}\right.$ for $30 \mathrm{~s}, 50^{\circ} \mathrm{C}$ for $30 \mathrm{~s}, 72^{\circ} \mathrm{C}$ for $120 \mathrm{~s}$ ) were performed (MJ Research thermocycler). A final extension was done at $72^{\circ} \mathrm{C}$ for $5 \mathrm{~min}$. PCR products were cleaved by EcoRI and HindIII, separated by polyacrylamide gel electrophoresis. The fragments of expected size were isolated from the gel and ligated into the EcoRI/HindIII-cleaved vector pBluescriptII SK(-). The recombinant constructs were transformed into the $\mathrm{DH} 5 \alpha$ strain of E. coli. Plasmid DNAs were isolated and both strands were sequenced with T7 Sequenase Version 2.0 DNA Sequencing Kit (USB) using T3 and T7 primers. Sequences were submitted to GenEMBL database under the following accession numbers: N. tabacum short family, AJ131161-AJ131165; N. tabacum long family, AJ131166-AJ131167; N. sylvestris, AJ131168-AJ131171; N. otophora, AJ131172-AJ131174; N. tomentosiformis, AJ131175-AJ131179.

\section{DNA preparation}

For most experiments, DNA was prepared from fresh young leaf tissues using standard procedures (SaghaiMaroof et al, 1984). The quantity and quality of DNA preparations were checked by absorbance at 260/280 nm. DNA of high molecular weight in agarose plugs for analysis by PFGE (Gene Navigator System, Pharmacia Biotech) was prepared using the modified protocol of Guidet et al (1990). Frozen leaf tissue was ground in liquid nitrogen to a fine powder using a mortar and pestle and transferred to 2.5 volumes of TEM ( $1 \mathrm{mM}$ Tris$\mathrm{HCl}$ pH 8.0; $50 \mathrm{mM}$ EDTA pH 8,0; $0.4 \mathrm{M}$ mannitol) at $50^{\circ} \mathrm{C}$, and the mixture quickly stirred. The same volume of $1.5 \%$ agarose (gelling point $28.5^{\circ} \mathrm{C}$ ) in TEM preheated to $50^{\circ} \mathrm{C}$ was added and the mixture stirred. The paste was transferred to a horizontally positioned plug mold (Bio-Rad) and chilled at $8^{\circ} \mathrm{C}$ for $20 \mathrm{~min}$. The plugs were transferred to tubes containing a six-fold excess of TES (10 mM Tris- $\mathrm{HCl}$ pH 8.0; 500 mM EDTA pH 8.0; 1.0\% Nlauroylsarcosine sodium salt) and incubated at $55^{\circ} \mathrm{C}$ for $60 \mathrm{~min}$ with one change of solution, and stored at $5^{\circ} \mathrm{C}$. Some plugs were transferred to TES with $0.5 \mathrm{mg} / \mathrm{ml}$ proteinase $\mathrm{K}$ and incubated at $55^{\circ} \mathrm{C}$ for 3 days, with one change of solution and washed five times, for $1 \mathrm{~h}$ each, in ET buffer (50 mM EDTA pH 8.0; $20 \mathrm{mM}$ Tris- $\mathrm{HCl} \mathrm{pH}$ 8.0 ) at $55^{\circ} \mathrm{C}$ (first wash) and room temperature (2-4 washes) with gentle agitation. Phenylmethylsulfonyl fluoride (PMSF) was included at a concentration of $1 \mathrm{mM}$ in the second and third wash. The plugs were stored in ET buffer at $5^{\circ} \mathrm{C}$. Size fractionation of high molecular weight DNA was carried out in $1 \%$ (w/v) agarose in $0.5 \times \mathrm{TBE}$ buffer for $24 \mathrm{~h}$ using PFGE with a time ramp of 50 to $120 \mathrm{~s}$ at $6 \mathrm{~V} / \mathrm{cm}$.

\section{Restriction endonuclease digestion}

Standard DNA preparations were digested with restriction enzymes using standard methods (Sambrook et al, 1988). For digestion of DNA for PFGE, agarose plugs were digested with $70-100$ units of enzyme per $50 \mu \mathrm{l}$ plug. The DNA was digested at $37^{\circ} \mathrm{C}$ for $16 \mathrm{~h}$ and then 30 units of fresh enzyme was added for another $4 \mathrm{~h}$.

\section{Distance tree analysis}

Multiple alignment of all sequenced $5 \mathrm{~S}$ rDNA spacers was done using the PileUp program (gap creation penalty 6, gap extension penalty 0 , Wisconsin Package ver.10.1, Genetics Computer Group (GCG), Madison, WI, USA) and edited by hand. A set of 100 distance matrices was calculated using the maximum likelihood method (ts $/$ tv ratio $=1.535$, WebPhylip, version 1.3., http:/ / sdmc.krdl.ord.sg:8080/ lxzhang/phylip). The neighbour-joining method and 'consense' program were used to generate a consensus unrooted distance tree.

\section{DNA probes}

The following 5S rDNA probes were used for FISH and Southern hybridization experiments:

(a) Genic 5S rDNA probe - the transcribed part of $5 S$ rDNA monomeric unit from N. tabacum (Fulneček et al, 1998; accession number AJ222659).

(b) T-genome sub-region probe - a GC-rich part of the long non-coding spacer of $5 S$ rDNA from N. tabacum (Figure 1).

\section{Southern blot hybridization}

Following electrophoresis, gels were blotted onto Hybond $\mathrm{N}+$ membranes and hybridized to ${ }^{32} \mathrm{P}$-labelled DNA probes $\left(>10^{8} \mathrm{dpm} / \mu \mathrm{g}\right.$ DNA, Fermentas, Dekaprime kit). Southern hybridization was carried out in $0.25 \mathrm{M}$ Na-phosphate buffer, $\mathrm{pH}$ 7.0, supplemented with $7 \%(\mathrm{w} / \mathrm{v})$ sodium dodecyl sulfate (SDS) at $65^{\circ} \mathrm{C}$ for $16 \mathrm{~h}$ followed by washing with $2 \times$ SSC $(1 \times$ SSC $=150 \mathrm{mM}$ $\mathrm{NaCl}, 15 \mathrm{mM} \mathrm{Na}$-citrate, $\mathrm{pH}$ 7.0), 0.1\% SDS (twice $5 \mathrm{~min}$ ), $0.2 \times$ SSC, $0.1 \%$ SDS (twice $15 \mathrm{~min}$ ). Membranes were exposed to X-ray film for 4 to $48 \mathrm{~h}$ (Sambrook et al, 1988) or visualized using a PhosphorImager (Storm, 
Molecular Dynamics) and quantified using ImageQuant software.

\section{In situ hybridization}

In situ hybridization was carried out as described in Leitch et al (1994) with modifications as described in Lim et al (2000b). Probes were labelled with digoxigenin-11 dUTP and detected with antidigoxigenin-FITC or with biotin-11-dUTP and detected with avidin-Cy3. Conditions of hybridization gave an estimated hybridization stringency of $80-85 \%$. Chromosomes and interphase nuclei from root tip meristematic cells were counterstained with $2 \mu \mathrm{g} / \mathrm{ml}$ DAPI (4',6-diamidino-2phenylindole).

\section{Results}

$5 S$ intergenic spacers in tobacco and progenitor species Using 5S rDNA sequence data (Fulneček et al, 1998), a pair of primers was designed to amplify the non-coding spacer sequences. PCR amplification of N. tabacum genomic DNA generated two prominent products of about 580 and $370 \mathrm{bp}$ suggesting two 5S rRNA gene families. In contrast there was one prominent band of about $580 \mathrm{bp}$ and a minor band of about $450 \mathrm{bp}$ from $N$. tomentosiformis and one band of about $370 \mathrm{bp}$ in N. sylvestris. PCR products were eluted from the gel and cloned. The following sequences were submitted to the EMBL database (accession numbers AJ131161-AJ131179): five short and two long $5 S$ spacer sequences from $N$. tabacum; four short $5 S$ spacers from $N$. sylvestris; three long $5 S$ spacers from $N$. otophora; and three long $5 S$ spacer sequences from $N$. tomentosiformis. The range in length of short spacers was 306-319 bp and the long spacers 509-527 bp. Three further 5S rDNA spacers were cloned from the low abundance variant in $N$. tomentosiformis, they ranged in size from $456-459 \mathrm{bp}$ and had a number of small deletions compared to the longer variant (Figure 1).

The average divergence between pairs of similar clones was expressed as percentage of nucleotide substitutions. The short spacer from $N$. tabacum was very similar to that of $N$. sylvestris (4\% divergence). The long spacer from $N$. tabacum was very similar to the spacer of $N$. tomentosiformis and N. otophora (4\% divergence). The sequence heterogeneity between individual clones of each family in tobacco also showed approximately $4 \%$ divergence. The sequences of the long and short spacer variants of $N$. tabacum 5 S units showed $80 \%$ sequence identity. The differences are largely attributed to a number of missing regions in the spacer of the short family (Figure 1). A phylogenetic scheme was constructed (Figure 2) using all the 5S non-coding spacer sequences cloned. These resolve into two well-supported groups; one contains the spacers found in $N$. sylvestris and the short spacers of $N$. tabacum (the S-genome family), and the other contains the spacers of $N$. tomentosiformis, $N$. otophora, and the long spacers of $N$. tabacum (T-genome family). The tree does not cluster the spacers into species groups as might be expected.

The $\mathrm{G}+\mathrm{C}$ content of the spacer was $38 \%$ for the $\mathrm{T}$ genome family and $34 \%$ for the S-genome family. A 130bp sequence, found only in the T-genome family clones (position 267-397 in Figure 1), had a higher 54\% G+C content and contained three GGCC motifs. This sequence is called here the T-genome sub-region, and specific pri-

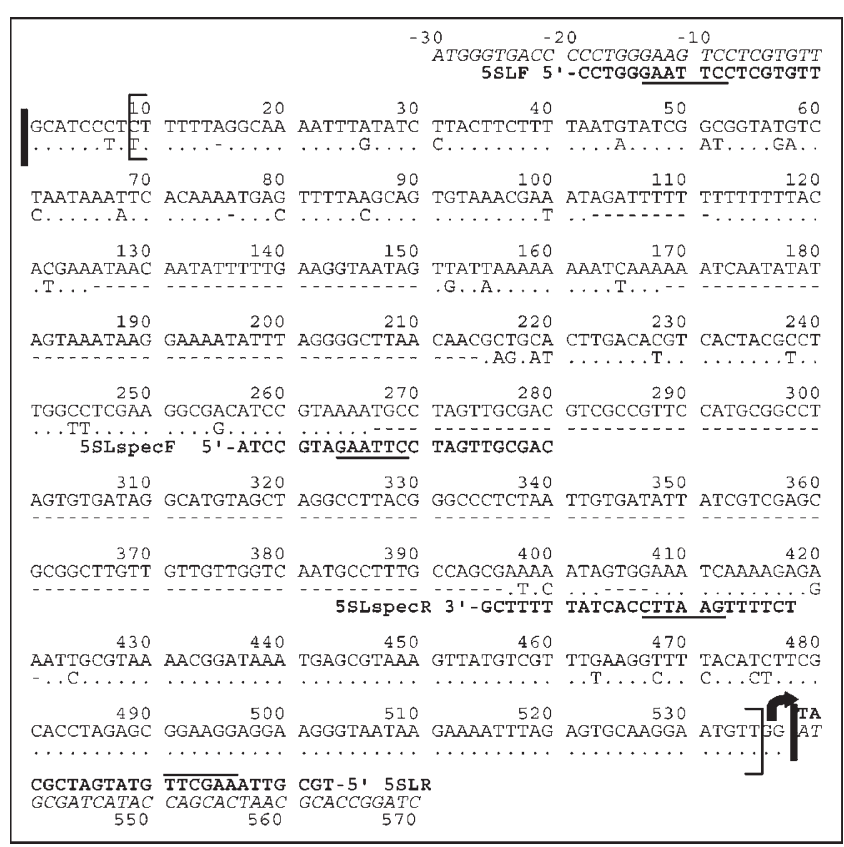

Figure 1 Sequence alignment of long (upper) and short (lower) Nicotiana tabacum 5S rDNA intergenic spacers (accession numbers AJ131166 and AJ131164; database names NTA131166 and NTA131164, respectively). The regions that correspond to these accession numbers are bordered by thick vertical lines. Sequences were obtained by PCR amplification of $N$. tabacum genomic DNA with 5SLF and 5SLR primers. The non-coding spacer sequences are in square brackets. The $\mathrm{T}$ genome sub-region is amplified with 5SLspecF and 5SLspecR occurring from 265-407 bp (accession number AJ131166). The curved arrow represents the start of transcription. Horizontal lines mark restriction enzyme sites.

mers were designed (Figure 1) for its amplification to generate a probe for further experiments (Figure 3 ). This probe hybridized only to the T-genome $5 S$ family of $N$. tabacum in Southern hybridisation experiments against $B s t$ NI digested genomic DNA (not shown).

The 5S spacer sequences revealed similarities with other plant spacers that also have a GC dinucleotide at -12 (with respect to the start site of transcription, Figure 1), a TATA-like motif at about -31 , and a long oligo-dT tract downstream from the transcribed region. At -1 from the start of transcription, there is frequently a C-nucleotide in other species (Wolters and Erdmann, 1988; Venkateswarlu et al, 1991), but this is substituted by a $\mathrm{T}$ nucleotide in most sequences cloned from $N$. sylvestris and the S-genome family of N. tabacum.

\section{Large-scale structure of $5 S$ rDNA arrays in N. tabacum}

PFGE mapping and in situ hybridization were used to determine the number, size and locations of $5 S$ rDNA arrays in the Nicotiana species. For PFGE, high molecular weight leaf DNA of $N$. tabacum cv. Vielblättriger was digested with several restriction endonucleases and hybridized sequentially with the probe against the $\mathrm{T}$ genome sub-region (absent in spacers of S-type units) and with the 5S rDNA genic probe (Figure 3). With each of $B c l$ I, HpaI and BglII DNA digestions, the T-genome subregion probe revealed one dominant band of approximately $500 \mathrm{~kb}$ in length suggesting that this is the length of the T-genome $5 S$ locus in the N. tabacum genome. No hybridization signal appeared after HaeIII digestion, 


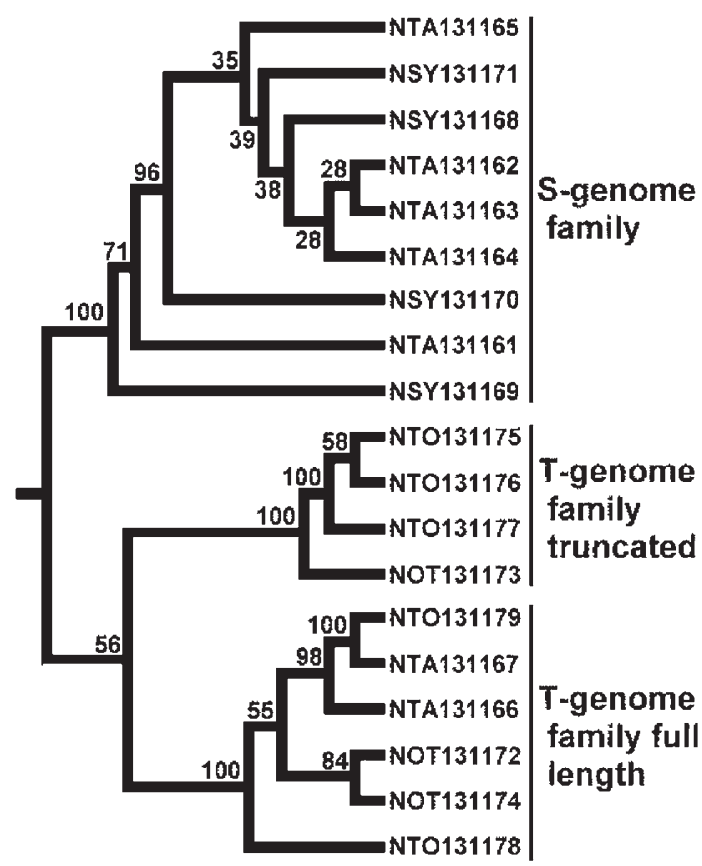

Figure 2 Consensus unrooted distance tree of 5S rDNA spacers from Nicotiana tabacum (NTA131161-67), N. tomentosiformis (NTO131175-79), N. otophora (NOT131172-74), and N. sylvestris (NSY131168-71). The names of spacers are as published in GenBank. The tree was generated from 100 bootstrapped multiple analyses (see methods). The numbers at the forks indicate the number of times the branch point occurred among the trees (out of 100). The tree is divided into two main groups corresponding to the Sgenome and T-genome $5 \mathrm{~S}$ rDNA families. The T-genome family is divided into two subgroups that cluster sequences according to spacer length.

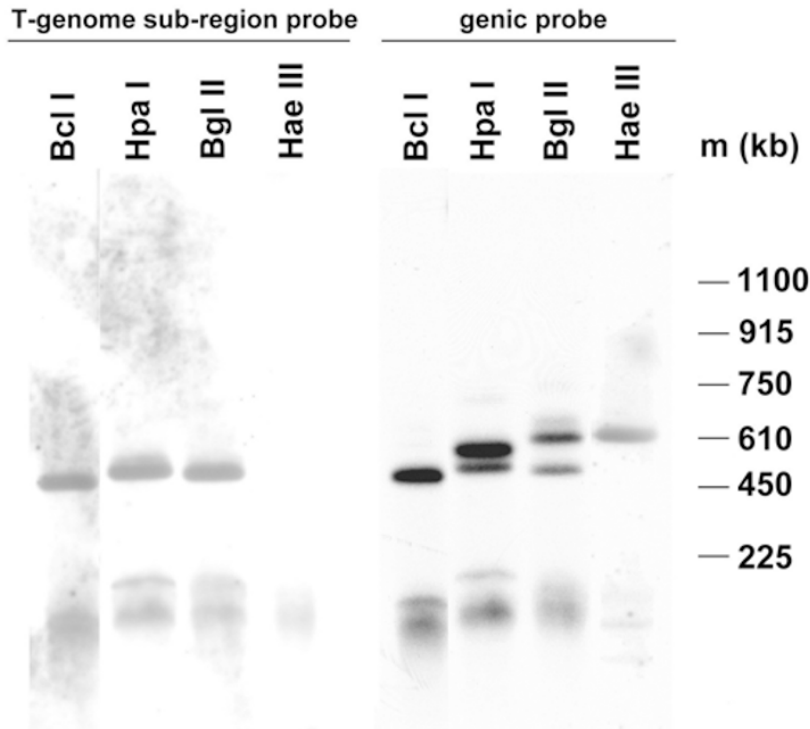

Figure 3 PFGE determination of the large scale organization of $5 S$ rDNA clusters in Nicotiana tabacum cv. Vielblättrigger. The restriction endonucleases used are indicated. The Southern blots were probed first with the T-genome sub-region probe, and then with the genic $5 \mathrm{~S}$ rDNA probe. $\mathrm{m}$, Saccharomyces cerevisiae size markers. probably because the T-genome $5 \mathrm{~S}$ units contain frequent HaeIII restriction sites and were digested to small fragments that migrated off the gel; these sites are absent in the non-coding spacer of the S-genome family (Figure 1). After rehybridization of the same blot with the genic probe, a $600 \mathrm{~kb}$ band appeared in HpaI, BglII and HaeIII digests. Since this band is absent with the T-genome subregion probe, the band must represent blocks of Sgenome derived units. The $600 \mathrm{~kb}$ band was not detected in the lane loaded with BclI-digested DNA probably because some, but not all, of the S-genome 5S units contained a BclI target site.

Previously, Ali et al (2000) detected two rDNA loci in $N$. tabacum and Lim et al (2000b) suggested that they occur on chromosomes S8 and T8. It was reasoned that using the T-genome sub-region probe it would be possible to localize each family to a particular chromosome and to determine the extent (if any) of mutual mixing. In $N$. tabacum two signals were obtained with the T-genome sub-region probe (yellow/green fluorescence) and four with the genic probe (red fluorescence) (Figure 4c-e). These results demonstrate that the $\mathrm{S}$ - and T-genome $5 \mathrm{~S}$ units occur in separate clusters at different chromosomal loci. In $N$. tomentosiformis and $N$. sylvestris, the genic probe labelled one locus only on chromosome 8 for each species respectively (Figure $4 \mathrm{a}, \mathrm{b}$ ). In $N$. tabacum it is already known that the two $5 \mathrm{~S}$ loci occur on $\mathrm{S}$ - and Tgenome chromosomes (Lim et al, 2000b), and it is reasonable to assume that the short family of $N$. sylvestris origin occur on chromosome S8 and the long $5 S$ family on chromosome T8 of $N$. tomentosiformis origin. An examination of several hundred root-tip meristematic interphase nuclei revealed no clustering of $5 S$ rDNA loci. Both Sand T- genome 5S loci showed signs of DNA decondensation, suggesting activity of units at both loci (Figure 4f).

\section{$5 S$ rDNA polymorphisms in tobacco cultivars}

Three widely used tobacco cultivars (Vielblättrigger, Petite Havana SR-1, and Samsun), two undifferentiated tobacco cell lines (TBY-2 and VBI-0), and the progenitor species of tobacco were analyzed for polymorphism in the lengths of the basic $5 S$ units. Southern hybridization of BstNI restricted DNAs showed the presence of both Tand S-genome $5 S$ families in all N. tabacum DNAs tested. However, differences were observed in the copy number of both families (Figure 5). The S-genome family was significantly reduced in both cell culture lines. This explains the strong in situ hybridization signal to only one locus in TBY-2 interphase nuclei (Figure $4 \mathrm{~g}, \mathrm{~h}$ ). The densitometric evaluation of radioactivity in individual bands was used to determine the copy number of monomers in individual families (Table 1).

\section{Discussion}

Two classes of 5S rDNA in the genome of $N$. tabacum Nicotiana tabacum is an allotetraploid with ancestors close to modern N. sylvestris (maternal donor of $S$ genome) and $N$. tomentosiformis (most probable paternal donor of $\mathrm{T}$ genome; cf. Goodspeed, 1954; Lim et al, 2000b). Two distinct families of $5 \mathrm{~S}$ rDNA have previously been localized in N. tabacum, a short $450 \mathrm{bp}$ family and a longer $630 \mathrm{bp}$ family (Fulneček et al, 1998). In this paper the two $5 S$ families have been localized to separate loci. Large-scale 

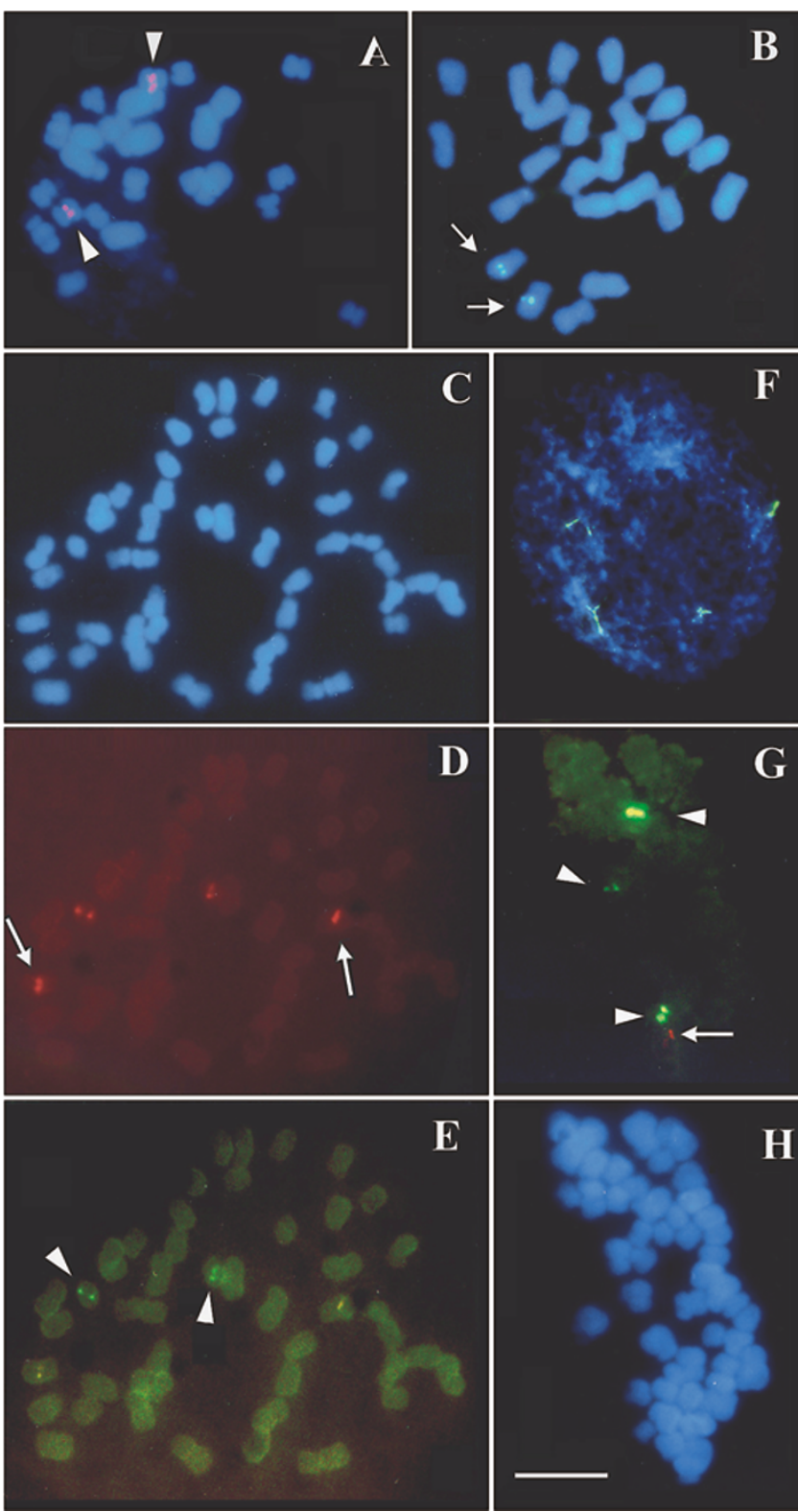

Figure 4 FISH using (a, b, f) $5 S$ gene probe detected with either FITC (yellow-green) or cy3 (red). (c-e, $\mathbf{g}, \mathbf{h}) 5 \mathrm{~S}$ gene probe (cy3) double-labelled with T-genome sub-region probe (FITC). Metaphases $(\mathbf{a}-\mathbf{e}, \mathbf{g}, \mathbf{h})$ and interphase nucleus (f) have been counterstained blue with DAPI. Sites of probe hybridization are arrowed, homologous sites carry the same type of arrow. (a) Nicotiana tomentosiformis; (b) N. sylvestris, (c-f) N. tabacum; (g, h) TBY-2, showing two large and one minor site with the T-genome subregion probe (arrow-head) and a minor site that is labelled only with the 5S genic probe (arrow). Scale bar $=10 \mu \mathrm{m}$.

mapping by pulsed field gel electrophoresis showed that both families form large homogenous blocks of tandem repeats of around $500 \mathrm{~kb}$ (T-genome family) and $600 \mathrm{~kb}$ (S-genome family) most probably located to chromosome T8 and chromosome S8, respectively (cf. also Lim et al, $2000 \mathrm{~b}$ ). The data revealed: (i) the length of basic unit of the S-genome family of tobacco is the same as the length of basic unit in N. sylvestris, likewise the length of basic unit of the T-genome family of tobacco is the same as the full length unit in N. tomentosiformis; (ii) the T-genome family of tobacco and $N$. tomentosiformis $5 S$ units have the

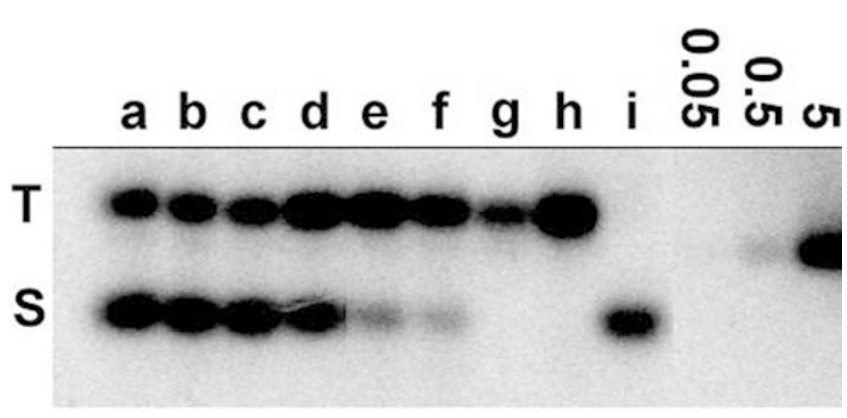

Figure 5 Determination of copy number of S- and T-genome 5S rDNA units in different cultivars of Nicotiana tabacum: (a) cv. Vielblättriger; (b) cv. Vielblättriger-callus; (c) cv. Samsun; (d) Petit Havana SR-1; (e) TBY-2 suspension culture; (f) VBI-0; and in the progenitor species (g) N. tomentosiformis; (h) N. otophora; and (i) N. sylvestris. $0.5 \mu \mathrm{g}$ Bst $\mathrm{NI}$ digested DNA was loaded in each lane. Lanes labelled $0.05,0.5$ and 5 contain respectively $0.05 \mathrm{ng}, 0.5 \mathrm{ng}$ and $5 \mathrm{ng}$ of recombinant pBluescript II SK(-) plasmid DNA carrying the $154 \mathrm{bp}$ fragment of $5 \mathrm{~S}$ rDNA gene from $N$. tabacum used as standards for quantitative evaluation of copy numbers.

Table 1 Estimated 5S rDNA unit copy numbers per haploid genome in S- and T-genome 5S families of Nicotiana species. Densitometric evaluation of Southern hybridization signal (Figure 5) was used for copy number determination. Error estimate is about $10 \%$

\begin{tabular}{llcr}
\hline Nicotiana species & \multicolumn{1}{c}{ Cultivar } & Copy number \\
\hline & & S-family & T-family \\
N. tabacum & Vielblättriger & 1100 & 900 \\
& Samsun & 1200 & 900 \\
& Petit Havana SR-1 & 950 & 1700 \\
& TBY-2 cell culture & 400 & 1600 \\
N. tomentosiformis & VBI-0 cell culture & 400 & 1600 \\
N. otophora & & 0 & 400 \\
N. sylvestris & & 0 & 1400 \\
& & 900 & 0 \\
\hline
\end{tabular}

same GC-rich sub-region that is absent in the S-genome family and N. sylvestris; and (iii) the non-coding spacer of S-genome family has high sequence similarity to the spacer of $N$. sylvestris while the non-coding spacer of Tgenome family has high sequence similarity to N. tomentosiformis. This organization of $5 S$ units in tobacco in which parental 5S units can be distinguished by length polymorphisms resembles other well-studied plant allopolyploids in the genera Triticum (Scoles et al, 1988) and Gossypium (Cronn et al, 1996).

\section{Evolution of $5 S$ repeats at the unit level}

It is informative that there is a similar diversity $(4 \%)$ of $5 S$ rDNA sequence structure: (i) among the T-genome units of tobacco; (ii) among the units of N. tomentosiformis and N. otophora; (iii) between these units and the T-family units of tobacco; (iv) among the S-genome units of tobacco; (v) among the units of N. sylvestris; and (vi) between these units and the S-genome units of tobacco. For this reason the phylogenetic reconstruction of $5 \mathrm{~S}$ units does not reflect a species phylogeny (Figure 2) and it suggests high sequence diversity in the progenitors of tobacco that are maintained in modern-day diploids and tobacco. Although only a subset of repeats was sequenced, it was not possible to detect any conserved base substitutions in the non-coding spacers of the S- and T-genome subunits 
compared with the diploid progenitor species. This is supported by extensive restriction analysis of numerous sites in both the non-coding (HaeIII and TaqI) and genic sequence (Bst NI and RsaI). These data suggest a slow nucleotide substitution and fixation rate of $5 S$ units in Nicotiana. It is reasonable to conclude that the time required for the fixation of a nucleotide change is longer than the 5-6 million years (Okamuro and Goldberg, 1985) since the formation of tobacco. This appears true not only for the genic region but also for the relatively rapidly evolving spacer sequence.

Sequence comparisons of S-and T-genome spacers revealed several interesting features. The region of high sequence identity is interrupted at three sites with insertions/deletions found in the diploid progenitors (Figure 1). The 130-bp T-genome sub-region has a distinct nucleotide composition which contrasts with the flanking sequences in being relatively GC-rich and forming a small 'GC-rich island'. The sequence could be an ancient transposition of non-5S DNA as happened in the 25S rRNA gene of Drosophila (Fedoroff, 1979), although it is too short to demonstrate any characteristic features of either transposons or retroelements. Alternatively, its origin could be a duplication of pre-existing short elements in the spacer region, and indeed, three GGCCT sites are observed. Sub-repeats are also found in the non-coding spacer of 5S rDNA of other plants (Scoles et al, 1988; Venkateswarlu et al, 1991; Singh et al, 1994) and perhaps amplification/reductions in sub-repeat numbers play a similar role in the evolution of 5S rDNA as they do for 18-26S rDNA (Flavell, 1989; Volkov et al, 1999).

\section{Evolution of $5 S$ repeats at the chromosome level}

Since the formation of $N$. tabacum there have been chromosomal interchanges (Kenton et al, 1993), amplification and diversification of GRD sequences (Ashby et al, 1997) and homogenization of 18-26S rDNA sequences via gene conversion (Volkov et al, 1999). Here data is presented suggesting that there is little or no change of parental 5S rDNA units in allopolyploid tobacco. Thus interlocus gene conversion has not occurred for $5 \mathrm{~S}$ rDNA as it did for 18-26S rDNA. Similarly, interlocus homogenisation at 18-26S rDNA, but not 5S rDNA, has been observed in allopolyploid Gossypium species (Cronn et al, 1996), suggesting similar evolutionary patterns.

There are several, although not necessarily exclusive, hypotheses to explain why 18-26S rDNA undergoes interlocus gene conversion while $5 \mathrm{~S}$ rDNA seems less vulnerable to this type of genetic modification.

(1) The length of mutual sequence homology and the presence of repetitive elements could facilitate genetic recombination. In 18-26S rDNA of both $N$. tomentosiformis and $N$. sylvestris, a significant part of the intergenic spacer is formed by short 10-15 bp sub-repeats some of which are conserved (Volkov et al, 1999). In contrast, the subrepeats of $5 S$ non-coding region are less abundant, highly degenerated and specific for either S- or T- genome units. Perhaps the length of mutual sequence homology, and the abundance of sub-repeats facilitates interlocus genetic recombination at meiosis or interphase in 18-26S rDNA, but not in $5 \mathrm{~S}$ rDNA.

(2) Cronn et al (1996) predicted that genetic recombination is influenced by chromosomal location. Perhaps sequences positioned near the telomere have a greater likelihood to interact with non-homologous chromosomes (eg, during end-to-end fusions) than interstitial or centromeric sequences. In both Gossypium and N. tabacum, the 18-26S rDNA loci which show interlocus homogenization are sub-telomeric, while $5 S$ repeats, which do not show this modification, are interstitial. Perhaps chromosomal position influences interlocus gene conversion rates, a hypothesis that could be tested in species where $5 S$ rDNA is located sub-telomerically, eg, in pea or wheat (Ellis et al, 1988).

(3) The interphase nucleus of plants is a highly structured entity showing domains of condensed and decondensed chromatin (Leitch, 2000). We argued in Lim et al (2000a) that gene conversion between homoeologous 18$26 \mathrm{~S}$ rDNA loci is most likely to occur during interphase of the cell cycle. It is at interphase that the different loci are decondensed, active and in close proximity within a nucleolus. Unlike 18-26S rDNA, the 5S sequences showed no such interphase associations, and were regularly well separated across the nucleus (Figure 4). This lack of interphase association may contribute to a lack of interlocus homogenization of the two 5S families in $N$. tabacum and elsewhere (Scoles et al, 1988; Cronn et al, 1996)

The copy number of $5 S$ repeats seems to be a characteristic feature of a given cultivar and is not influenced by environmental or developmental factors (plants and derived calluses had essentially the same number of repeats; data not shown). Interestingly, a decrease in the copy number in one $5 S$ rDNA family in tobacco is often associated with an increase in copy number in the second family suggesting that a mechanism maintaining a relatively fixed number of $5 S$ repeats may be active. It would be interesting to analyze other stocks of $N$. tomentosiformis from different wild populations to determine if they have variable $5 S$ rDNA copy numbers which could explain the polymorphisms in N. tabacum. Differences in copy number between cultivars has already been reported for $5 \mathrm{~S}$ rDNA in wheat (Roder et al, 1992) and soybean (Danna et al, 1996). Contractions or expansions of an array seem to be frequent genetic events in the evolution of $5 S$ repeats.

\section{Acknowledgements}

We thank the Grant Agency of the Czech Republic (grant numbers 204/98/0191, 204/01/0313, 204/99/D001 and $521 / 01 / 0037)$, the Natural Environment Research Council, and The Royal Society for support. The hardware and the GCG software has been acquired with the help of the grant LB98210 of the Ministry of Education of the Czech Republic Program of research and development 'Promotion of information infrastructure for research and development'. B Koukalová is thanked for valuable assistance. We thank Ms L Murad, Professor CP Lichtenstein and Dr Nichols for their help.

\section{References}

Ali HBM, Fransz P, Shubert I (2000). Localization of 5S RNA genes on tobacco chromosomes. Chromosome Res 8: 85-87.

Ashby MK, Warry A, Bejarano ER, Khashoggi A, Burrell M, Lichtenstein CP (1997). Analysis of multiple copies of geminiviral DNA in the genome of four closely related Nicotiana 
species suggest a unique integration event. Plant Mol Biol 35: 313-321.

Cronn RC, Zhao X, Paterson AH, Wendel JF (1996). Polymorphism and concerted evolution in a tandemly repeated gene family: 5S ribosomal DNA in diploid and allopolyploid cottons. J Mol Evol 42: 685-705.

Danna KJ, Workman R, Coryell V, Keim P (1996). 5S r RNA genes in tribe Phaseolae: array size, number, and dynamics. Genome 39: 445-455.

Ellis TH, Lee D, Thomas CM, Simpson PR, Cleary WG, Newman MA et al (1988). 5S rRNA genes in Pisum: sequence, longrange and chromosomal organization. Mol Gen Genet 214: 333-342.

Fedoroff NV (1979). On spacers. Cell 16: 697-710.

Flavell RB (1989). Variation in structure and expression of ribosomal DNA loci in wheat. Genome 31: 963-968.

Fulneček J, Matyášek R, Kovař́k A, Bezdĕk M (1998). Mapping of 5-methylcytosine residues in Nicotiana tabacum 5S rRNA genes by genomic sequencing. Mol Gen Genet 259: 133-141.

Goodspeed TH (1954). The genus Nicotiana. Chronica Botanica, Waltham, Massachusetts.

Guidet F, Rogowsky P, Langridge P (1990). A rapid method of preparing megabase plant DNA. Nucleic Acids Res 18: 4955.

Kenton A, Parokonny AS, Gleba YY, Bennett MD (1993). Characterization of the Nicotiana tabacum L. genome by molecular cytogenetics. Mol Gen Genet 240: 159-169.

Kovařík A, Fajkus J, Koukalová B, Bezdĕk M (1996). Speciesspecific evolution of telomeric and rDNA repeats in the tobacco composite genome. Theor Appl Genet 92 1108-1111.

Leitch AR, Schwarzacher T, Leitch IJ, Jackson D (1994). In Situ Hybridisation: A Practical Approach. Bios Scientific Publishers: Oxford.

Leitch AR (2000). Higher levels of organization in the interphase nucleus of cycling and differentiated cells. Microbiol Mol Biol Rev 64: 138-152.

Lim KY, Kovařík A, Matyášek R, Bezdĕk M, Lichtenstein CP, Leitch AR (2000a). Gene conversion of ribosomal DNA in Nicotiana tabacum is associated with undermethylated, decondensed and probably active gene units. Chromosoma 109 $161-172$.

Lim KY, Matyášek R, Lichtenstein CP, Leitch AR (2000b). Molecular cytogenetic analyses and phylogenetic studies in the Nicotiana section Tomentosae. Chromosoma 109: 245-258.

Moscone EA, Matzke MA, Matzke AJ (1996). The use of combined FISH/GISH in conjunction with DAPI counterstaining to identify chromosomes containing transgene inserts in amphidiploid tobacco. Chromosoma 105: 231-236.

Nagata T, Nemoto Y, Hasezawa S (1992). Tobacco BY-2 cell line as a "HeLa" cell in the cell biology of higher plants. Int Rev Cytol 132: 1-30.

Okamuro JK, Goldberg RB (1985). Tobacco single-copy DNA is highly homologous to sequences present in the genomes of its diploid progenitors. Mol Gen Genet 198: 290-298.

Opatrný Y, Opatrná J (1976). The specificity of the effect of 2,4$\mathrm{D}$ and NAA of the growth, micromorphology, and occurrence of starch in long-term Nicotiana tabacum L. cell strains. Biologia Plantarum 18: 359-365.

Parokonny AS, Kenton AY (1995). Comparative physical mapping and evolution of the Nicotiana tabacum L. karyotype, In: Brandham PE, Bennett MD (eds) Kew Chromosome Conference $I V$, Royal Botanic Gardens: Kew.

Roder MS, Sorrells ME, Tanksley SD (1992). 5S ribosomal gene clusters in wheat: pulsed field gel electrophoresis reveals a high degree of polymorphism. Mol Gen Genet 232: 215-220.

Saghai-Maroof MA, Soliman KM, Jorgensen RA, Allard RW (1984) Ribosomal DNA spacer-length polymorphisms in barley: mendelian inheritance, chromosomal location, and population dynamics. Proc Natl Acad Sci USA 81: 8014-8018.

Sambrook J, Fritsch EF, Maniatis T (1988). Molecular Cloning: A Laboratory Manual. Cold Spring Harbor Laboratory Press: New York.

Scoles CJ, Gill BSZ, Xin Y, Clarke BC, McIntyre CL, Chapman $C$ et al (1988). Frequent duplication and deletion events in the 5S RNA genes and the associated spacer regions of the Triticeae. Plant Syst Evol 160: 105-122.

Singh K, Bhatia S, Lakshmikumaran M (1994). Novel variants of the 5S rRNA genes in Eruca sativa. Genome 37: 121-128.

Sperisen C, Ruals J, Meins F (1991). Comparison of cloned genes provides evidence for intergenomic exchange of DNA in the evolution of a tobacco glucan endo-1,3-beta-glucosidase gene family. Proc Natl Acad Sci USA 88: 1820-1824.

Venkateswarlu K, Lee SW, Nazar RN (1991). Conserved upstream sequence elements in plant 5S ribosomal RNAencoding genes. Gene 105: 249-254.

Volkov RA, Borisjuk NV, Panchuk II, Schweizer D, Hemleben V (1999). Elimination and rearrangement of parental rDNA in the allotetraploid Nicotiana tabacum. Mol Biol Evol 16: 311-320.

Wolters J, Erdmann VA (1988). Cladistic analysis of ribosomal RNAs-the phylogeny of eukaryotes with respect to the endosymbiotic theory. Biosystems 21: 209-214. 